\title{
Coding Theorems for Turbo Code Ensembles
}

\author{
Hui Jin and Robert J. McEliece, Fellow, IEEE
}

\author{
Invited Paper
}

\begin{abstract}
This paper is devoted to a Shannon-theoretic study of turbo codes. We prove that ensembles of parallel and serial turbo codes are "good" in the following sense. For a turbo code ensemble defined by a fixed set of component codes (subject only to mild necessary restrictions), there exists a positive number $\gamma_{0}$ such that for any binary-input memoryless channel whose Bhattacharyya noise parameter is less than $\gamma_{0}$, the average maximum-likelihood (ML) decoder block error probability approaches zero, at least as fast as $n^{-\beta}$, where $\beta$ is the "interleaver gain" exponent defined by Benedetto et al. in 1996.
\end{abstract}

Index Terms-Bhattacharyya parameter, coding theorems, maximum-likelihood decoding (MLD), turbo codes, union bound.

\section{INTRODUCTION}

$\mathbf{T}$ HE invention of turbo codes in 1993 [6], and the explosion of research that followed, has revolutionized every aspect of channel coding. Turbo codes appear to offer nothing less than a solution to the challenge issued by Shannon in 1948 [33]: to devise practical methods of communicating reliably at rates near channel capacity. And while there has been a good deal of excellent theoretical work on turbo codes, it seems fair to say that practice still leads theory by a considerable margin. In particular, there has been little previous Shannon-theoretic work on turbo codes. By "Shannon-theoretic" we mean a study of the average performance of the codes in the turbo-code ensemble under maximum-likelihood decoding (MLD). Of course, there is little possibility that MLD of turbo codes can be implemented practically, but since the turbo decoding algorithm seems to be, in most cases, a close approximation to MLD, it is important to know the MLD potential for this class of codes. In any case, this paper is devoted to a Shannon-theoretic study of turbo codes. In particular, it may be viewed as an elaboration of the following remark, which was made in [24]:

"The presence [in turbo-codes] of the pseudorandom interleavers between the component codes ensures that the resulting overall code behaves very much like a long random code, and by Shannon's theorems, a long random code is likely to be 'good'..."

Manuscript received April 17, 2001; revised September 17, 2001. This work was supported by the NSF under Grant CCR-9804793, and under grants from Sony, Qualcomm, and Caltech's Lee Center for Advanced Networking.

H. Jin was with the Department of Electrical Engineering, California Institute of Technology, Pasadena, CA 91125 USA. He is now with Flarion Technologies, Inc., Bedminster, NJ 07921 USA (e-mail: h.jin@ flarion.com).

R. J. McEliece is with the Department of Electrical Engineering, California Institute of Technology, Pasadena, CA 91125 USA (e-mail: rjm@systems.caltech.edu).

Communicated by S. Shamai, Guest Editor.

Publisher Item Identifier S 0018-9448(02)04013-0.
In this paper, we will prove that turbo codes are indeed good, in the following sense. For any turbo code ensemble, parallel or serial, defined by a fixed set of component codes (subject only to mild necessary restrictions), there exists a positive number $\gamma_{0}$, such that on any binary-input memoryless channel whose Bhattacharyya noise parameter is less than $\gamma_{0}$, the average maximum-likelihood (ML) decoder block ${ }^{1}$ error probability approaches zero, at least as fast as $n^{-\beta}$, where $\beta$ is the (ensemble-dependent) "interleaver gain" exponent defined by [2]-[5]. (For an exact statement of these results, see Section VIII, Theorems 8.1 and 8.4.) It is only fair to acknowledge that similar results were first stated, and proved informally, by Benedetto et al., in [2]-[5].

Here is an outline of the paper.

- Section II: A definition of the parallel and serial turbo-code ensembles.

- Section III: A discussion of general code ensembles, and their weight enumerators.

- Section IV: The Bhattacharyya noise parameter and the union bound, for binary input discrete memoryless channels.

- Section V: A coding theorem for general code ensembles, combining the ensemble weight enumerator with the union bound.

- Section VI: Estimates (upper bounds) of the weight enumerators of the parallel turbo code ensembles defined in Section II.

- Section VII: Estimates (upper bounds) of the weight enumerators of the serial turbo code ensembles defined in Section II.

- Section VIII: Statement and proof of the main results.

- Section IX: Examples: The CCSDS ensemble, and the ensemble of RA codes.

- Section X: Discussion and conclusions.

- Appendix A: Combinatorial facts about convolutional codes.

- Appendix B: Some useful inequalities.

- Appendix C: Extension of main theorems to bit error probability.

\footnotetext{
${ }^{1}$ Later, we will also consider bit error probability, but for now let us agree that "good" refers to vanishingly small block error probability.
} 


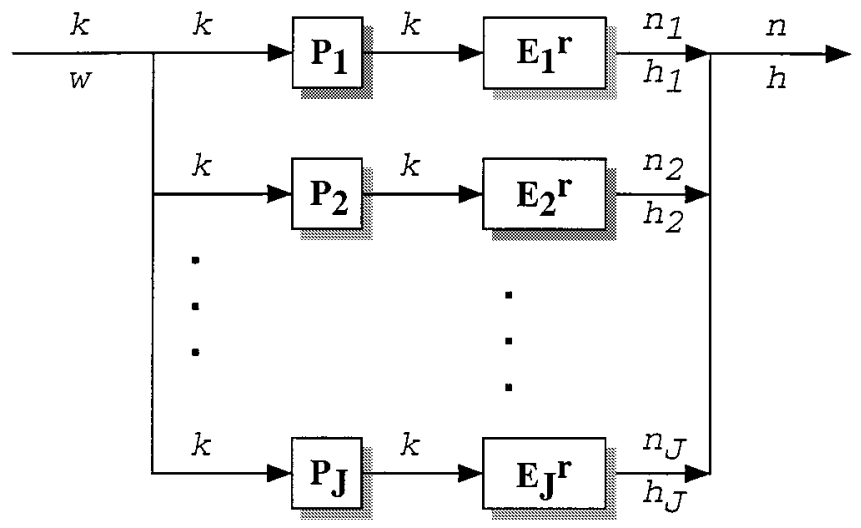

Fig. 1. Encoder for a parallel turbo code. The numbers above the input-output lines indicate the length of the corresponding block, and those below the lines indicate (when present) the Hamming weight of the block.

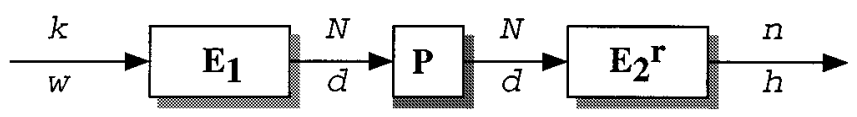

Fig. 2. Encoder for a serial turbo code. As in Fig. 1, the numbers above the input-output lines indicate the length of the corresponding block, and those below the lines indicate the Hamming weight of the block.

\section{TURBo-CODE ENSEMBLES}

The general structure of a parallel turbo code is shown in Fig. 1. There are $J$ interleavers (pseudorandom permutations) $P_{1}, P_{2}, \ldots, P_{J}$ and $J$ recursive convolutional encoders $E_{1}^{r}, E_{2}^{r}, \ldots, E_{J}^{r}{ }^{2}{ }^{2}$ An information block of length $k$ is permuted by interleaver $P_{i}$ and then encoded (and truncated) by $E_{i}^{r}$, producing a codeword of length $n_{i}$, for $i=1,2, \ldots, J$. These $J$ codewords are then sent to the channel. The overall code is therefore an $(n, k)$ linear block code, with $n=\sum_{i=1}^{J} n_{i}$. If $R_{i}=k / n_{i}$ is the rate of the $i$ th component code $C_{i}$, then the overall code rate is easily seen to be $R=\left(\sum_{i=1}^{J} R_{i}^{-1}\right)^{-1}$. Because there are $k !$ choices for each interleaver, ${ }^{3}$ there are a large number of codes with the structure shown in Fig. 1. We call this set of codes the $\left[E_{1}^{r}\left\|E_{2}^{r}\right\| \cdots \| E_{J}^{r}\right]$ ensemble. (We will define a code ensemble more precisely in Section III.)

Our first main result (Theorem 8.1) implies that if $J \geq 2$, the $\left[E_{1}^{r}\left\|E_{2}^{r}\right\| \cdots \| E_{J}^{r}\right]$ ensemble is "good," in the sense defined in Section I.

A serial turbo code has the general structure shown in Fig. 2. An information block of length $k$ is encoded by an outer encoder $E_{1}$ into a codeword of length $N$, which is permuted by an interleaver $P$, and then encoded by a recursive inner encoder $E_{2}^{r}$ into a codeword of length $n$. The outer code $C_{1}$ is a truncated convolutional code, ${ }^{4}$ and the inner code $C_{2}$ is a truncated recursive convolutional code. The overall code is therefore an $(n, k)$ linear block code, with rate $R=R_{1} R_{2}$, where $R_{1}$ is the rate of the outer code and $R_{2}$ is the rate of the inner code. Because of

\footnotetext{
${ }^{2}$ Here and hereafter, a superscript " $r$ " indicates that the designated encoder is recursive, i.e., any input of weight 1 produces an output of infinite weight.

${ }^{3}$ Without loss of generality, we may assume that $P_{1}$ is the identity permutation, so that there are really only $J-1$ interleavers.

${ }^{4}$ We note that a block code can be viewed as a convolutional code without memory, so that $E_{1}$ may be a block encoder.
}

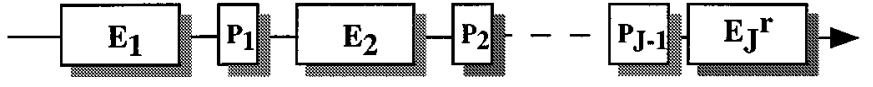

Fig. 3. Encoder for a multiple serial turbo code.

the choices for the interleaver, there are $N$ ! codes with the structure shown in Fig. 2. We call this set of codes the $\left[E_{1} \Rightarrow E_{2}^{r}\right]$ ensemble.

Our second main result (Theorem 8.4) implies that if the minimum distance of the outer code $C_{1}$ is at least three, the $\left[E_{1} \Rightarrow E_{2}^{r}\right]$ ensemble is also "good."

Finally, we mention the "multiple" serial turbo code depicted in Fig. 3. Here, an information block of length $k$ is encoded by a first encoder $E_{1}$ into a first codeword of length $N_{1}$, which is permuted by an interleaver $P_{1}$; this codeword is then encoded by a second encoder $E_{2}$ into a second codeword of length $N_{2}$, which is permuted by an interleaver $P_{2}$, etc. This process is repeated $J$ times, concluding with the $J$ th encoder $E_{J}^{r}$, which is required to be recursive. The overall code rate is $R=R_{1} R_{2} \cdots R_{J}$, where $R_{i}$ is the rate of $E_{i}$. We call this set of codes the $\left[E_{1} \Rightarrow\right.$ $\left.E_{2} \Rightarrow \cdots \Rightarrow E_{J}^{r}\right]$ ensemble. Our third main theorem (Theorem 8.7), which is stated without proof, guarantees that the $\left[E_{1} \Rightarrow E_{2} \Rightarrow \cdots \Rightarrow E_{J}^{r}\right]$ ensemble is good whenever

$$
\sum_{i=1}^{J-2} d_{i}+\left\lceil d_{J-1}|i|\right\rceil \geq J
$$

\section{Code Ensembles, In General}

Parallel and serial turbo codes are important examples of code ensembles, but our results can be applied to other ensembles as well. In this section, we will give a general definition of a code ensemble.

By an ensemble of linear codes, then, we mean a sequence $\mathcal{C}_{n_{1}}, \mathcal{C}_{n_{2}}, \ldots$ of sets of linear codes, where $\mathcal{C}_{n_{i}}$ is a set of $\left(n_{i}, k_{i}\right)$ codes with common rate $R_{i}=k_{i} / n_{i}$. We assume that the sequence $n_{1}, n_{2}, \ldots$ approaches infinity, and that

$$
\lim _{i \rightarrow \infty} R_{i}=R
$$

where $R$ is called the rate of the ensemble.

We shall be concerned with the weight structure of the ensemble, and with this in mind we introduce some notation. If $C$ is an $(n, k)$ linear code, we denote its weight enumerator by the list $A_{0}(C), A_{1}(C), \ldots, A_{n}(C)$. In other words, $A_{h}(C)$ is the number of words of weight $h$ in $C$, for $h=0,1, \ldots, n$. When no ambiguity is likely to occur, we denote the weight enumerator simply by $A_{0}, A_{1}, \ldots, A_{n}$. We will also need the cumulative weight enumerator

$$
A_{\leq h}=\sum_{d=1}^{h} A_{d}, \quad \text { for } h=1, \ldots, n .
$$

In words, $A_{\leq h}$ is the number of nonzero codewords of weight $\leq h$.

When the code $C$ is viewed as the set of possible outputs of a particular encoder $E$, we denote by $A_{w, h}^{(E)}$ the number of $(\boldsymbol{x}, \boldsymbol{y})$ pairs where the encoder input $\boldsymbol{x}$ has weight $w$ and the corresponding encoder output $\boldsymbol{y}$ (codeword) has weight $h$. Usually the encoder will be understood, and the simpler notation $A_{w, h}$ will do. The set of numbers $A_{w, h}$ is called 
the input-output weight enumerator (IOWE) for the code. In analogy with (3.1), we define the cumulative input-output weight enumerator (CIOWE)

$$
A_{w, \leq h}=\sum_{d=1}^{h} A_{w, d} .
$$

Returning now to the ensemble, we define the average weight enumerator for the set $\mathcal{C}_{n}$ as the list

$$
\bar{A}_{0}^{(n)}, \bar{A}_{1}^{(n)}, \ldots, \bar{A}_{n}^{(n)}
$$

where

$$
\bar{A}_{h}^{(n)} \triangleq \frac{1}{\left|\mathcal{C}_{n}\right|} \sum_{C \in \mathcal{C}_{n 2}} A_{h}(C), \quad \text { for } h=0,1, \ldots, n .
$$

Similarly, we define the average cumulative weight enumerator $\bar{A}_{\leq h}^{(n)}$, the average IOWE $\bar{A}_{w, h}^{(n)}$, and the average CIOWE $\bar{A}_{w,<h}^{(n)}$.

For each $n$ in the sequence $n_{1}, n_{2}, \ldots$, the $n$th spectral shape function is defined as

$$
r_{n}(\delta) \triangleq \frac{1}{n} \log \bar{A}_{\lfloor\delta n]}^{(n)}, \quad \text { for } 0<\delta<1 .
$$

Thus $\bar{A}_{h}^{(n)}=e^{n r_{n}(\delta)}$, where $\delta=h / n$.

Finally, we define the asymptotic spectral shape

$$
r(\delta) \triangleq \lim _{n \rightarrow \infty} r_{n}(\delta), \quad \text { for } 0<\delta<1
$$

provided the limit exists. In this case, we can say, roughly, that for large $n$, if the ratio $\delta=h / n$ is fixed, then

$$
\bar{A}_{h}^{(n)} \sim e^{n r(\delta)} .
$$

It is worth noting here that the main difficulty in proving our main results (Theorems 8.1 and 8.4) is that we are unable to compute $r(\delta)$ for the $\left[E_{1}^{r}\left\|E_{2}^{r}\right\| \cdots \| E_{J}^{r}\right]$ and $\left[E_{1} \Rightarrow E_{2}^{r}\right]$ ensembles. Instead, we have had to resort to upper bounds on $r(\delta)$ (see (6.8) and (7.8)), based on the work of Kahale and Urbanke [21], which render our results existence theorems only.

\section{MEMORYLESS BinARY-INPUT ChANNELS AND THE UNION BOUND}

Since turbo codes, as we have defined them, are binary codes, we consider using them on memoryless binary input channels. Such a channel has binary input alphabet $\{0,1\}$ and arbitrary output alphabet $\Omega$. If the channel input is a binary random variable $X$, then the channel output is a random variable $Y$. If $\Omega$ is finite, then $Y$ is characterized by transition probabilities $p(y \mid 0)$, $p(y \mid 1)$, i.e., for $y \in \Omega$

$$
\begin{aligned}
& p(y \mid 0)=\operatorname{Pr}\{Y=y \mid X=0\} \\
& p(y \mid 1)=\operatorname{Pr}\{Y=y \mid X=1\} .
\end{aligned}
$$

If $\Omega$ is a subset of $R^{r}$, where $R$ is the real line, then $Y$ is characterized by transition probability densities $p(y \mid 0), p(y \mid 1)$, i.e., if $S$ is a measurable subset of $\Omega$

$$
\begin{aligned}
& \int_{S} p(y \mid 0) d y=\operatorname{Pr}\{Y \in S \mid X=0\} \\
& \int_{S} p(y \mid 1) d y=\operatorname{Pr}\{Y \in S \mid X=1\} .
\end{aligned}
$$

The "noisiness" of the channel can be summarized by the Bhattacharyya noise parameter $\gamma$, which is defined by

$$
\gamma=\sum_{y \in \Omega} \sqrt{p(y \mid 0) p(y \mid 1)}
$$

if $\Omega$ is finite and

$$
\gamma=\int_{\Omega} \sqrt{p(y \mid 0) p(y \mid 1)} d y
$$

if $\Omega=R^{r}$. It is easy to see (by the Cauchy-Schwarz inequality) that $\gamma \leq 1$ with equality if and only if $p(y \mid 0)=p(y \mid 1)$ for all $y$, in which case the channel has capacity zero. ${ }^{5}$

For example, for a binary erasure channel with erasure probability $p$, we have

$$
\gamma_{\mathrm{BEC}}=p
$$

For a binary-symmetric channel (BSC) with crossover probability $p$ we have

$$
\gamma_{\mathrm{BSC}}=2 \sqrt{p(1-p)}
$$

For the asymmetric " $Z$ " channel, we have

$$
\gamma_{Z}=\sqrt{p}
$$

For an additive Gaussian channel with $\Omega=R$ and

$$
\begin{aligned}
& p(y \mid 0)=\frac{1}{\sqrt{2 \pi \sigma^{2}}} e^{-(y-1)^{2} / 2 \sigma^{2}} \\
& p(y \mid 1)=\frac{1}{\sqrt{2 \pi \sigma^{2}}} e^{-(y+1)^{2} / 2 \sigma^{2}}
\end{aligned}
$$

a short calculation using (4.2) gives

$$
\gamma_{\mathrm{AGC}}=e^{-1 / 2 \sigma^{2}}
$$

As a final example, for the binary input coherent Rayleighfading channel with perfect channel state information available to the receiver, we have $\Omega=R \times R^{+}$, and for $(y, a) \in \Omega$

$$
\begin{aligned}
& p(y, a \mid 0)=\frac{1}{\sqrt{2 \pi \sigma^{2}}} e^{-(y-a)^{2} / 2 \sigma^{2}} 2 a e^{-a^{2}} \\
& p(y, a \mid 1)=\frac{1}{\sqrt{2 \pi \sigma^{2}}} e^{-(y+a)^{2} / 2 \sigma^{2}} 2 a e^{-a^{2}}
\end{aligned}
$$

In this case, (4.2) yields

$$
\gamma_{\mathrm{RF}, \mathrm{CSI}}=\left(1+\frac{1}{2 \sigma^{2}}\right)^{-1}
$$

The importance of $\gamma$ is that $\gamma^{h}$ is an upper bound on the ML decoder error probability for a binary code with two codewords separated by a Hamming distance of $h$ (see [23, Theorem 7.5]). It follows that for an $(n, k)$ binary linear code with $A_{h}$ codewords of weight $h$, we have the following upper bound, usually

\footnotetext{
${ }^{5}$ The so-called cutoff rate for the channel is $R_{0}=1-\log _{2}(1+\gamma)$, which is positive if and only if the capacity is positive, i.e., $\gamma<1$.
} 
called the union bound, on the ML decoder word error probability:

$$
\begin{aligned}
P_{W} & \leq \sum_{h=1}^{n} A_{h} \gamma^{h} \\
& =\sum_{h=1}^{n} A_{h} e^{-\alpha h}
\end{aligned}
$$

where $\alpha=-\log \gamma \geq 0$ is what we shall call the noise exponent for the channel. Since, as noted previously, $\gamma \leq 1$, we have $\alpha \geq 0$ with equality if and only if the channel has zero capacity. Similarly, we can use the union bound to estimate the ML decoder bit error probability

$$
P_{b} \leq \sum_{h=1}^{n} \sum_{w=1}^{k} \frac{w}{k} A_{w, h} \gamma^{h}
$$

where $A_{w, k}$ is the input-output weight enumerator of the code.

Since the union bound is linear on weight enumerators, it also applies to ensembles of codes, with $A_{h}$ replaced by $\bar{A}_{h}^{(n)}$, the average number of codewords of weight $h$ in $\mathcal{C}_{n}$

$$
\begin{aligned}
\bar{P}_{W}^{(n)} & \leq \sum_{h=1}^{n} \bar{A}_{h}^{(n)} e^{-\alpha h} \\
& =\sum_{h=1}^{n} e^{-n\left(\alpha \delta-r_{n}(\delta)\right)}
\end{aligned}
$$

where in (4.5) $\delta=h / n$. For the ensemble bit error probability we have correspondingly

$$
\bar{P}_{b}^{(n)} \leq \sum_{h=1}^{n} \sum_{w=1}^{k} \frac{w}{k} \bar{A}_{w, h}^{(n)} e^{-\alpha h} .
$$

\section{A CODING THEOREM}

In this section, by combining the spectral shape function with the union bound, we obtain an upper bound on the ML decoder word error probability for an ensemble of binary linear codes (Theorem 5.1). It shows that under certain conditions, there exists a threshold $c_{0}$ such that if the channel noise exponent $\alpha$ exceeds $c_{0}$, the ensemble word error probability approaches 0 . We shall see that the low-weight codewords in the ensemble determine whether or not the threshold $c_{0}$ is finite.

To begin, we introduce some notation. First, let $D_{n}$ be a fixed sequence of integers satisfying

$$
\begin{aligned}
\frac{D_{n}}{n^{\epsilon}} & \rightarrow 0, \quad \text { for all } \epsilon>0 \\
\frac{\log n}{D_{n}} & \rightarrow 0 .
\end{aligned}
$$

For example, $D_{n}=\log ^{2} n$ will do. Second, we define the noise thresholds for the ensemble

$$
\begin{gathered}
c_{0}^{(n)} \triangleq \sup _{D_{n} / n<\delta \leq 1} r_{n}(\delta) / \delta \\
c_{0} \triangleq \limsup _{n \rightarrow \infty} c_{0}^{(n)} .
\end{gathered}
$$

Finally, the $n$th innominate sum is defined as follows:

$$
Z^{(n)}(D) \triangleq \sum_{h=1}^{D} \bar{A}_{h}^{(n)}
$$

where $D$ is an integer with $1 \leq D \leq n$. In words, $Z^{(n)}(D)$ is the average number of words of weight $\leq D$ for a code in the set $C_{n}$. (Incidentally, it is also an upper bound on the probability that the minimum distance of a code in $C_{n}$ is $\leq D$.)

Theorem 5.1: Suppose the ensemble threshold $c_{0}$ defined in (5.4) is finite, and the channel error exponent $\alpha$ satisfies $\alpha>c_{0}$. Then, if $\bar{P}_{W}^{(n)}$ denotes the ensemble ML decoder error probability, there exists an integer $n_{0}$ and positive constants $K$ and $\epsilon$ such that for $n>n_{0}$

$$
\bar{P}_{W}^{(n)} \leq Z^{(n)}\left(D_{n}\right)+K e^{-\epsilon D_{n}} .
$$
we have

Proof: Since the channel error exponent $\alpha$ is nonnegative,

$$
A_{h} e^{-\alpha h} \leq A_{h} .
$$

Therefore, by (4.4) and (4.5)

$$
\begin{aligned}
\bar{P}_{W}^{(n)} & \leq \sum_{h=1}^{D_{n}} \bar{A}_{h}^{(n)}+\sum_{h>D_{n}} \bar{A}_{h}^{(n)} e^{-\alpha h} \\
& =Z^{(n)}\left(D_{n}\right)+\sum_{h>D_{n}} e^{-h\left(\alpha-r_{n}(\delta) / \delta\right)} .
\end{aligned}
$$

If $\alpha>c_{0}$, then there exists an integer $n_{0}$, and an $\epsilon>0$ such that for $n>n_{0}, \alpha-c_{0}^{(n)}>\epsilon$. Hence, for $n>n_{0}$ and $h>D_{n}$, we have

$$
\alpha-\frac{r_{n}(\delta)}{\delta} \geq \alpha-c_{0}^{(n)}>\epsilon
$$

so that

$$
e^{-h\left(\alpha-r_{n}(\delta) / \delta\right)} \leq e^{-h \epsilon}
$$

Thus,

$$
\sum_{h>D_{n}} e^{-h\left(\alpha-r_{n n}(\delta) / \delta\right)} \leq \sum_{h>D_{n}} e^{-h \epsilon}=K e^{-D_{n} \epsilon}
$$

where $K=e^{-\epsilon} /\left(1-e^{-\epsilon}\right)$. Substituting (5.8) into (5.6), we have (5.5).

Corollary 5.2: If, in addition, $Z^{(n)}\left(D_{n}\right)=O\left(n^{-\beta}\right)$ where $\beta>0$, then for $\alpha>c_{0}$

$$
P_{W}^{(n)}=O\left(n^{-\beta}\right) .
$$

Proof: Note that $n^{-\beta}=e^{-\beta \log n}$. The result now follows from (5.5) and (5.2).

The question as to whether $c_{0}$ is finite is partially answered by the following two technical results.

Theorem 5.3: For a code ensemble $\mathcal{C}$, the code threshold $c_{0}$ is finite if and only if for all sequences $\epsilon_{n}$ such that $\epsilon_{n}>D_{n} / n$ and $\epsilon_{n} \rightarrow 0$

$$
c_{0}^{\prime}=\lim _{n \rightarrow \infty} \sup _{D_{n} / n<\delta<\epsilon_{n 2}} r_{n}(\delta) / \delta
$$

is finite.

Proof: Clearly

$$
\sup _{D_{n} / n<\delta<\epsilon_{n}} \frac{r_{n}(\delta)}{\delta} \leq \sup _{D_{n} / n<\delta \leq 1} \frac{r_{n}(\delta)}{\delta}
$$

so that if $c_{0}$ as defined in (5.3) is finite, so is $c_{0}^{\prime}$, for any choice of $\epsilon_{n}$. 
To complete the proof, we will show that if $c_{0}^{\prime}$ is finite, so is $c_{0}$, or rather the contrapositive, i.e., $c_{0}=\infty$ implies $c_{0}^{\prime}=\infty$. If $c_{0}$ is infinite, then there is a convergent subsequence $\delta_{n} \rightarrow \delta_{0}$ such that $D_{n} / n<\delta_{n} \leq 1$ with

$$
\lim _{n \rightarrow \infty} \frac{r_{n}\left(\delta_{n}\right)}{\delta_{n}}=\infty
$$

If $\delta_{0}>0$, note that ${ }^{6}$

$$
\bar{A}_{h}^{(n)} \leq\left(\begin{array}{l}
n \\
h
\end{array}\right) \leq e^{n H(\delta)}
$$

hence $r_{n}(\delta)=\log \bar{A}_{h}^{(n)} / n \leq H(\delta)$. Thus,

$$
\lim _{n \rightarrow \infty} \frac{r_{n}\left(\delta_{n}\right)}{\delta_{n}} \leq \frac{H\left(\delta_{0}\right)}{\delta_{0}}
$$

which contradicts (5.11). Thus, $\delta_{0}=0$. Hence, if we define $\epsilon_{n}=\min \left(2 \delta_{n}, 1\right)$, we have

$$
\sup _{D_{n} / n<\delta<\epsilon_{n}} \frac{r_{n}(\delta)}{\delta} \geq \frac{r_{n}\left(\delta_{n}\right)}{\delta_{n}} \rightarrow \infty .
$$

Thus, (5.11) diverges, which shows that $c_{0}^{\prime}$ is infinite.

Corollary 5.4: If there exists a function $s(\delta)$ and constants $\gamma_{n}=O\left(D_{n} / n\right)$ such that $r_{n}(\delta) \leq \gamma_{n}+s(\delta)$ for all sufficiently small $\delta$ and all sufficiently large $n$, then the ensemble noise threshold $c_{0}$ is finite provided

$$
\limsup _{\delta \rightarrow 0} \frac{s(\delta)}{\delta}<\infty
$$

Proof: We use Theorem 5.3. Thus, let $\epsilon_{n}$ be a sequence such that $\epsilon_{n}>D_{n} / n$ and $\epsilon_{n} \rightarrow 0$. Then

$$
\begin{aligned}
& \lim _{n \rightarrow \infty} \sup _{D_{n} / n<\delta<\epsilon_{n}} r_{n}(\delta) / \delta \\
& \leq \lim _{n \rightarrow \infty} \sup _{D_{n} / n<\delta<\epsilon_{n}}\left(\gamma_{n}+s(\delta)\right) / \delta \\
& \leq \limsup _{n \rightarrow \infty}\left(n \gamma_{n} / D_{n}\right)+\lim _{n \rightarrow \infty}\left(\sup _{0<\delta<\epsilon_{n}} s(\delta) / \delta\right) \\
& \leq K+\limsup _{\delta \rightarrow 0} s(\delta) / \delta<\infty \text {. }
\end{aligned}
$$

Thus by Theorem 5.3, the code threshold $c_{0}$ is finite.

\section{WEIGHT ENUMERATOR ESTIMATES FOR PARALLEL TURBO CODE ENSEMBLES}

For the $\left[E_{1}^{r}\left\|E_{2}^{r}\right\| \cdots \| E_{J}^{r}\right]$ ensemble, the average IOWE can be obtained from the IOWEs of the component codes using the "uniform interleaver" technique [2]

$$
\bar{A}_{w, h}^{(n)}=\frac{1}{\left(\begin{array}{l}
k \\
w
\end{array}\right)^{J-1}} \sum_{\sum_{i=1}^{J} h_{i}=h} \prod_{i=1}^{J} A_{w, h_{i}}^{[i]}
$$

where $A_{w, h_{i}}^{[i]}$ is the IOWE for the $i$ th component code $C_{i}$ (see Fig. 1 for notation). Therefore,

$$
\begin{aligned}
\bar{A}_{w, \leq h}^{(n)} & =\frac{1}{\left(\begin{array}{c}
k \\
w
\end{array}\right)^{J-1}} \sum_{\sum_{i=1}^{J} h_{i} \leq h} \prod_{i=1}^{J} A_{w, h_{i}}^{[i]} \\
& \leq \frac{1}{\left(\begin{array}{c}
k \\
w
\end{array}\right)^{J-1}} \sum_{h_{1}=1}^{h} \cdots \sum_{h_{J}=1}^{h} \prod_{i=1}^{j} A_{w, h_{i}}^{[i]}
\end{aligned}
$$

${ }^{6} \mathrm{We}$ have collected several useful inequalities on binomial coefficients in Appendix B.

$$
\begin{aligned}
& =\frac{1}{\left(\begin{array}{c}
k \\
w
\end{array}\right)^{J-1}} \prod_{i=1}^{J}\left(\sum_{h_{i}=1}^{h} A_{w, h_{i}}^{[i]}\right) \\
& =\frac{\prod_{i=1}^{J} A_{w, \leq h}^{[i]}}{\left(\begin{array}{c}
k \\
w
\end{array}\right)^{J-1}} .
\end{aligned}
$$

Next, we apply the bound of Theorem A.3 from Appendix A to each $A_{w, \leq h}^{[i]}$ in (6.2). The truncation length for each $C_{i}$ is less than its code length $n_{i}$, which, in turn, is strictly less than $n=\sum_{i} n_{i}$. Defining $\eta=\max _{i} \eta_{i}$, and noting that the binomial coefficient $\left(\begin{array}{l}n \\ j\end{array}\right)$ is an increasing function of $n$, we obtain

$$
A_{w, \leq h}^{[i]} \leq \theta_{i}^{w} \sum_{j=0}^{\lfloor w / 2\rfloor}\left(\begin{array}{c}
n \\
j
\end{array}\right)\left(\begin{array}{c}
\eta h \\
w-j
\end{array}\right) .
$$

If $\eta h<n$, then by Proposition B.1, $\left(\begin{array}{c}n \\ j\end{array}\right)\left(\begin{array}{c}\eta h \\ w-j\end{array}\right)$ attains its maximum for $0 \leq j \leq\lfloor w / 2\rfloor$ at $j=\lfloor w / 2\rfloor$. Thus (provided $h<n / \eta)$, each $A_{w, \leq h}^{[i]}$ can be bounded as follows:

$$
\begin{aligned}
& A_{w, \leq h}^{[i]} \leq \theta_{i}^{w}\left(\left\lfloor\frac{w}{2}\right\rfloor+1\right)\left(\begin{array}{c}
n \\
\lfloor w / 2\rfloor
\end{array}\right)\left(\begin{array}{c}
\eta h \\
\lceil w / 2\rceil
\end{array}\right) \\
& \leq\left(2 \theta_{i}\right)^{w}\left(\begin{array}{c}
n \\
\lfloor w / 2\rfloor
\end{array}\right)\left(\begin{array}{c}
\eta h \\
\lceil w / 2\rceil
\end{array}\right) \\
&\text { since } \left.\lfloor w / 2\rfloor+1 \leq 2^{w}\right) .
\end{aligned}
$$

Combining (6.2) and (6.4), we obtain

$$
\bar{A}_{w, \leq h}^{(n)} \leq \theta^{w} \frac{\left(\begin{array}{c}
n \\
\lfloor w / 2\rfloor
\end{array}\right)^{J}\left(\begin{array}{c}
\eta h \\
\lceil w / 2\rceil
\end{array}\right)}{\left(\begin{array}{l}
k \\
w
\end{array}\right)^{J-1}}
$$

for some constant $\theta>1$. Consequently, for small $\delta, \bar{A}_{\leq h}^{(n)}$ can be upper-bounded as follows:

$$
\begin{aligned}
\bar{A}_{\leq h}^{(n)} & \leq \sum_{w=1}^{\mu h} A_{w, \leq h}^{(n)} \\
& \leq \sum_{w=1}^{\mu h} \theta^{w} \frac{\left(\begin{array}{c}
n \\
\lfloor w / 2\rfloor
\end{array}\right)^{J}\left(\begin{array}{c}
\eta h \\
\lceil w / 2\rceil
\end{array}\right)}{\left(\begin{array}{c}
k \\
w
\end{array}\right)} .
\end{aligned}
$$

(The sum in (6.5) stops at $\mu h$ rather than $k$ because of Theorem A.1). Equation (6.5) will be used to bound the innominate sum $Z^{(n)}\left(D_{n}\right)$ that appears in Theorem 5.1.

To bound $r_{n}(\delta)$ for small $\delta$, we simplify (6.5), by replacing the summation with the maximum term times the number of terms. Since $\left(\begin{array}{c}\eta h \\ l\end{array}\right)<2^{\eta h}$ for any integer $l$, and $\mu h \leq n$, we have

$$
\bar{A}_{\leq h}^{(n)} \leq n 2^{J \eta h} \theta^{\mu h} \max _{1 \leq w \leq \mu h} \frac{\left(\begin{array}{c}
n \\
\lfloor w / 2\rfloor
\end{array}\right)^{J}}{\left(\begin{array}{c}
k \\
w
\end{array}\right)^{J-1}} .
$$

Using the inequalities in (B3), we have

$$
\begin{aligned}
\frac{\left(\begin{array}{c}
n \\
\lfloor w / 2\rfloor
\end{array}\right)^{J}}{\left(\begin{array}{c}
k \\
w
\end{array}\right)^{J-1}} & \leq \frac{e^{n J H(x / 2)}}{e^{n R(J-1) H(x / R)}}(k+1)^{J-1} \\
& \leq \frac{e^{n J H(x / 2)}}{e^{n R(J-1) H(x / R)}} n^{J-1}
\end{aligned}
$$


where $R=k / n$ (the rate of the overall code), and $x=w / n$. Combining (3.4) with (6.6) and (6.7), we have

$$
\begin{aligned}
r_{n}(\delta)= & \frac{1}{n} \log \bar{A}_{h}^{(n)} \\
\leq & \frac{1}{n} \log \bar{A}_{\leq h}^{(n)} \\
\leq & J \frac{\log n}{n}+T \delta \\
& +\sup _{0<x \leq \mu \delta}\left\{J H\left(\frac{x}{2}\right)-R(J-1) H\left(\frac{x}{R}\right)\right\}
\end{aligned}
$$

where $T$ is a constant. Equation (6.8) will be used with Theorem 5.4 to prove that $c_{0}$ is finite for the $\left[E_{1}^{r}\|\cdots\| E_{J}^{r}\right]$ ensemble.

\section{Weight ENUMERATOR ESTIMATES FOR SERIAL TURBo CODE ENSEMBLES}

For the $\left[E_{1} \Rightarrow E_{2}^{r}\right]$ ensemble, the average IOWE can be obtained from the weight enumerator of the outer code $C_{1}$ and the IOWE of the inner code $C_{2}$ [4] (see Fig. 2 for notation)

$$
\bar{A}_{h}^{(n)}=\sum_{d=1}^{N} \frac{A_{d}^{[1]} A_{d, h}^{[2]}}{\left(\begin{array}{l}
N \\
d
\end{array}\right)} .
$$

Hence

$$
\bar{A}_{\leq h}^{(n)}=\sum_{d=1}^{N} \frac{A_{d}^{[1]} A_{d, \leq h}^{[2]}}{\left(\begin{array}{l}
N \\
d
\end{array}\right)} .
$$

Since if $A_{d, h}^{[2]} \neq 0, d$ is less than $\mu h$ by Theorem A.1 (where $\mu=\mu\left(E_{2}\right)$ ), applying Theorem A.2 to the outer code $C_{1}$ and Theorem A.3 to the inner code $C_{2}$ with $L_{1}$ as the trellis length for $C_{1}$ and $L_{2}$ as the trellis length for $C_{2}$, we obtain

$$
\begin{aligned}
\bar{A}_{\leq h}^{(n)} & =\sum_{d=1}^{\mu h} \frac{A_{d}^{[1]} A_{d, \leq h}^{[2]}}{\left(\begin{array}{c}
N \\
d
\end{array}\right)} \\
& \leq \sum_{d=1}^{\mu h} \theta^{d} \frac{\left(\begin{array}{c}
L_{1} \\
\left\lfloor d / d_{1}\right.
\end{array}\right)}{\left(\begin{array}{c}
N \\
d
\end{array}\right)} \sum_{j=0}^{\lfloor d / 2\rfloor}\left(\begin{array}{c}
L_{2} \\
j
\end{array}\right)\left(\begin{array}{c}
\eta h \\
d-j
\end{array}\right)
\end{aligned}
$$

where $d_{1}$ is the free distance of $C_{1}$. If $C_{1}$ is an $\left(n_{1}, k_{1}, m_{1}\right)$ code of rate $R_{1}=k_{1} / n_{1}$, and $C_{2}$ is an $\left(n_{2}, k_{2}, m_{2}\right)$ code with rate $R_{2}=k_{2} / n_{2}$, then we have $L_{1}=N / n_{1}, L_{2}=n / n_{2}$, and $N=R_{2} n$, so that

$$
\begin{aligned}
L_{1} & =\frac{k_{2}}{n_{1} n_{2}} n=\alpha n \\
N & =\frac{k_{2}}{n_{2}} n=\beta n \\
L_{2} & =\frac{1}{n_{2}} n=\gamma n
\end{aligned}
$$

where $\alpha=k_{2} / n_{1} n_{2}, \beta=k_{2} / n_{2}$, and $\gamma=1 / n_{2}$. Thus, (7.3) becomes

$$
\bar{A}_{\leq h}^{(n)} \leq \sum_{d=1}^{\mu h} \theta^{d} \frac{\left(\begin{array}{c}
\alpha n \\
\left\lfloor d / d_{1}\right\rfloor
\end{array}\right)}{\left(\begin{array}{c}
\beta n \\
d
\end{array}\right)} \sum_{j=0}^{\lfloor d / 2\rfloor}\left(\begin{array}{c}
\gamma n \\
j
\end{array}\right)\left(\begin{array}{c}
\eta h \\
d-j
\end{array}\right) .
$$

For $\delta=h / n$ small enough, we have $\eta h=\eta \delta n<n$, hence

$$
\left(\begin{array}{c}
\gamma n \\
j
\end{array}\right)\left(\begin{array}{c}
\eta h \\
d-j
\end{array}\right) \leq\left(\begin{array}{c}
\gamma n \\
\lfloor d / 2\rfloor
\end{array}\right)\left(\begin{array}{c}
\eta h \\
\lceil d / 2\rceil
\end{array}\right)
$$

for any $0 \leq j \leq\lfloor d / 2\rfloor$ by Proposition B.1. Therefore, replacing the inner sum in (7.4) with $\lfloor d / 2\rfloor+1$ times the right-hand side of (7.5), we have

$$
\begin{aligned}
\bar{A}_{\leq h}^{(n)} & \leq \sum_{d=1}^{\mu h} \theta^{d} \frac{\left(\begin{array}{c}
\alpha n \\
\left\lfloor d / d_{1}\right\rfloor
\end{array}\right)}{\left(\begin{array}{c}
\beta n \\
n
\end{array}\right)}(\lfloor d / 2\rfloor+1)\left(\begin{array}{c}
\gamma n \\
\lfloor d / 2\rfloor
\end{array}\right)\left(\begin{array}{c}
\eta h \\
\lceil d / 2\rceil
\end{array}\right) \\
& \leq \sum_{d=1}^{\mu h}\left(2 \theta_{1}\right)^{d} \frac{\left(\begin{array}{c}
\alpha n \\
\left\lfloor h / d_{1}\right\rfloor
\end{array}\right)}{\left(\begin{array}{c}
\beta n \\
d
\end{array}\right)}\left(\begin{array}{c}
\gamma n \\
\lfloor d / 2\rfloor
\end{array}\right)\left(\begin{array}{c}
\eta h \\
\lceil d / 2\rceil
\end{array}\right) .
\end{aligned}
$$

(The last inequality because $\lfloor d / 2\rfloor+1 \leq 2^{d}$.) The inequality (7.6) will be used to bound the innominate sum $Z^{(n)}\left(D_{n}\right)$.

To bound $r_{n}(\delta)$, we further simplify (7.6). Using the inequality $\left(\begin{array}{c}\eta h \\ l\end{array}\right)<2^{\eta h}$, and bounding the summation in (7.6) by the number of terms times the maximum term, we have

$$
\bar{A}_{\leq h}^{(n)} \leq n \theta^{\mu h} 2^{\eta h} \max _{1 \leq d \leq \mu h}\left(\begin{array}{c}
\alpha n \\
\left\lfloor h / d_{1}\right\rfloor
\end{array}\right)\left(\begin{array}{c}
\gamma n \\
\lfloor d / 2\rfloor
\end{array}\right) /\left(\begin{array}{c}
\beta n \\
d
\end{array}\right) .
$$

Using techniques like those that led from (6.6) to (6.8), the spectral shape can thus be upper-bounded by the following expression, where $x=d / n$ :

$$
\begin{aligned}
r_{n}(\delta) \leq 2 \frac{\log n}{n}+T \delta+\sup _{0<x \leq \mu \delta}\{\alpha H & \left(\frac{x}{d_{1} \alpha}\right)+\gamma H\left(\frac{x}{2 \gamma}\right) \\
& \left.-\beta H\left(\frac{x}{\beta}\right)\right\}
\end{aligned}
$$

where $T$ is a constant. Equation (7.8) will used with Corollary 5.4 to prove that $c_{0}$ is finite for the $\left[E_{1} \Rightarrow E_{2}^{r}\right]$ ensemble.

\section{PROOF OF MAIN Results}

In this section, we give the proofs of our main results, viz. Theorems 8.1 and 8.4. These theorems first appeared as conjectures, implicitly in [2] and [4] and explicitly in [12]. Theorem 8.1 can be summarized, using the language of [2] and [4], by saying that the $\left[E_{1}^{r}\|\cdots\| E_{J}^{r}\right]$ ensemble has word error probability interleaving gain exponent $-J+2$, and bit error probability interleaving gain exponent $-J+1$. Theorem 8.4 can be summarized by saying that the $\left[E_{1} \Rightarrow E_{2}^{r}\right]$ ensemble has word error probability interleaving gain exponent $-\left\lfloor\frac{d_{1}-1}{2}\right\rfloor$, and bit error probability interleaving gain exponent $-\left\lfloor\frac{d_{1}+1}{2}\right\rfloor$, where $d_{1}$ is the minimum distance of the outer code $C_{1}$.

Theorem 8.1: For the $\left[E_{1}^{r}\|\cdots\| E_{J}^{r}\right]$ ensemble, if $J \geq 2$, there exists a positive number $c_{0}$, such that for any binary-input memoryless channel whose noise exponent $\alpha$ satisfies $\alpha>c_{0}$, we have

$$
\begin{aligned}
& \bar{P}_{W}^{(n)}=O\left(n^{-J+2+\epsilon}\right) \\
& \bar{P}_{b}^{(n)}=O\left(n^{-J+1+\epsilon}\right)
\end{aligned}
$$

for any $\epsilon>0$.

Proof: (We restrict our attention to the statement about $\bar{P}_{W}^{(n)}$. The extension to $\bar{P}_{b}^{(n)}$ is explained in Appendix C.) Given Theorem 5.1 and Corollary 5.2, it will be sufficient to prove the following two lemmas.

Lemma 8.2: For the $\left[E_{1}^{r}\|\cdots\| E_{J}^{r}\right]$ ensemble, if $J \geq 2, c_{0}$ is finite. 
Proof: We use Corollary 5.4, with the upper bound (6.8) on the code spectral shape

$$
\begin{aligned}
\gamma_{n} & =\frac{J \log n}{n} \\
s(\delta) & =T \delta+\sup _{0<x \leq \mu \delta}\left(J H\left(\frac{x}{2}\right)-R(J-1) H\left(\frac{x}{R}\right)\right) .
\end{aligned}
$$

To show that $\limsup s(\delta) / \delta<\infty$, we need to show that the following limit is finite:

$$
\lim _{\delta \rightarrow 0} \frac{1}{\delta} \sup _{0<x<\mu \delta}\left(J H\left(\frac{x}{2}\right)-R(J-1) H\left(\frac{x}{R}\right)\right) .
$$

But by Proposition B.3, this is true, since $J / 2-R(J-1) / R=$ $-J / 2+1 \leq 0$, for $J \geq 2$.

Lemma 8.3: For the $\left[E_{1}^{r}\|\cdots\| E_{J}^{r}\right]$ ensemble, if $J \geq 2$

$$
Z^{(n)}\left(D_{n}\right)=O\left(n^{-J+2+\epsilon}\right)
$$

for any positive $\epsilon$.

Proof: Using the upper bound (6.5) on $\bar{A}_{\leq h}^{(n)}$, we have

$$
\begin{aligned}
Z^{(n)}\left(D_{n}\right) & =\sum_{h=1}^{D_{n}} \bar{A}_{n}^{(n)}=\bar{A}_{\leq D_{n}}^{(n)} \\
& \leq \sum_{w=1}^{\mu D_{n}} \theta^{w} \frac{\left(\begin{array}{c}
n \\
\lfloor w / 2\rfloor
\end{array}\right)^{J}\left(\begin{array}{c}
\eta D_{n} \\
\lceil w / 2\rceil
\end{array}\right)}{\left(\begin{array}{c}
R n \\
w
\end{array}\right)^{J-1}} \\
& \stackrel{\text { (a) }}{\leq} \sum_{w=1}^{\mu D_{n}} \Theta^{w} n^{J\lfloor w / 2\rfloor-(J-1) w} D_{n}^{(2 J-1) w} \\
& \stackrel{(\mathrm{b})}{=} O\left(n^{-J+2+\epsilon}\right) .
\end{aligned}
$$

In (a), we have used the following inequalities (see (B2)):

$$
\begin{aligned}
\left(\begin{array}{c}
n \\
\lfloor w / 2\rfloor
\end{array}\right) & \leq n^{\lfloor w / 2\rfloor} \\
\left(\begin{array}{c}
\eta D_{n} \\
\lceil w / 2\rceil
\end{array}\right) & \leq\left(\eta D_{n}\right)^{\lceil w / 2\rceil}<\left(\eta D_{n}\right)^{w} \\
\left(\begin{array}{c}
R n \\
w
\end{array}\right) & \geq(R n)^{w} / w^{w} \geq(R n)^{w} /\left(\mu D_{n}\right)^{w} .
\end{aligned}
$$

Here, $\Theta$ represents a new constant. For (b), the sum in (8.1) can be upper-bounded by $\mu D_{n}$ times the largest term, which, by Proposition B.2, is the $w=2$ term for large enough $n$. Notice $D_{n}=o\left(n^{\epsilon}\right)$ for any positive $\epsilon$ by (5.1).

Next, we prove the corresponding theorem for serial turbo codes.

Theorem 8.4: For the $\left[E_{1} \Rightarrow E_{2}^{r}\right]$ ensemble, if the free distance of the outer code satisfies $d_{1} \geq 3$, there exists a positive number $c_{0}$ such that for any binary-input memoryless channel whose noise exponent $\alpha$ satisfies $\alpha>c_{0}$

$$
\begin{aligned}
& \bar{P}_{W}^{(n)}=O\left(n^{-\left\lfloor\frac{d_{1}-1}{2}\right\rfloor+\epsilon}\right) \\
& \bar{P}_{b}^{(n)}=O\left(n^{-\left\lfloor\frac{d_{1}+1}{2}\right\rfloor+\epsilon}\right)
\end{aligned}
$$

for arbitrary $\epsilon>0$.

(We again restrict our attention to $\bar{P}_{W}^{(n)}$, leaving $\bar{P}_{W}^{(n)}$ to Appendix C.) Again, because of Theorem 5.1 and Corollary 5.2, it is sufficient to prove the following two lemmas.
Lemma 8.5: For the $\left[E_{1} \Rightarrow E_{2}^{r}\right]$ ensemble, if the free distance of the outer code satisfies $d_{1} \geq 2, c_{0}$ is finite.

Proof: Corollary 5.4, together with (7.8), makes it sufficient to show

$\lim _{\delta \rightarrow 0} \frac{1}{\delta} \sup _{0<x<\mu \delta}\left(a H\left(\frac{x}{d_{1} \alpha}\right)+\gamma H\left(\frac{x}{2 \gamma}\right)-\beta H\left(\frac{x}{\beta}\right)\right)<\infty$.

But by Proposition B.3, this is true, since $1 / d_{1}+1 / 2-1 \leq 0$, for $d_{1} \geq 2$.

Lemma 8.6: For the $\left[E_{1} \Rightarrow E_{2}^{r}\right]$ ensemble, if $d_{1} \geq 3$

$$
Z^{(n)}\left(D_{n}\right)=O\left(n^{-\left\lfloor\frac{d_{1}-1}{2}\right\rfloor+\epsilon}\right)
$$

for arbitrary $\epsilon>0$.

Proof: With the bound (7.6), we have

$$
\begin{aligned}
Z^{(n)}\left(D_{n}\right) & =\bar{A}_{\leq D_{n}}^{(n)} \\
& \leq \sum_{d=d_{1}}^{\mu D_{n 2}} \theta \eta^{d} \frac{\left(\begin{array}{c}
\alpha n \\
\left\lfloor d / d_{1}\right\rfloor
\end{array}\right)}{\left(\begin{array}{c}
\beta n \\
d
\end{array}\right)}\left(\begin{array}{c}
n \\
\lfloor d / 2\rfloor
\end{array}\right)\left(\begin{array}{c}
\eta D_{n} \\
\lceil d / 2\rceil
\end{array}\right) \\
& \stackrel{\text { (a) }}{\leq} \sum_{d=d_{1}}^{\mu D_{n 2}} \Theta^{d_{n}\left\lfloor d / d_{1}\right\rfloor-\lceil d / 2\rceil} D_{n}^{d+\lceil d / 2\rceil} \\
& \stackrel{\text { (b) }}{=} O\left(n^{-\left\lfloor\frac{d_{1}-1}{2}\right\rfloor+\epsilon}\right) .
\end{aligned}
$$

In step (a), we have used the following inequalities \{ see (B2)):

$$
\begin{aligned}
\left(\begin{array}{c}
\alpha n \\
\left\lfloor d / d_{1}\right\rfloor
\end{array}\right) & \leq(\alpha n)^{\left\lfloor d / d_{1}\right\rfloor} \\
\left(\begin{array}{c}
\beta n \\
d
\end{array}\right) & \geq(\beta n)^{d} / d^{d} \geq(\beta n)^{d} /\left(\mu D_{n}\right)^{d} \\
\left(\begin{array}{c}
n \\
\lfloor d / 2\rfloor
\end{array}\right) & \leq n^{\lfloor d / 2\rfloor}
\end{aligned}
$$

and

$$
\left(\begin{array}{c}
\eta D_{n} \\
\lceil d / 2\rceil
\end{array}\right) \leq\left(\eta D_{n}\right)^{\lceil d / 2\rceil} .
$$

For step (b), the sum is upper-bounded by $\mu D_{n}$ times the biggest term, which by Proposition B.2 is the $d=d_{1}$ term, as $n$ becomes large. The conclusion follows, since $D_{n}=o\left(n^{\epsilon}\right)$ for any positive $\epsilon$.

We conclude this section by stating a theorem, without proof, about the $\left[E_{1} \Rightarrow E_{2} \Rightarrow \cdots \Rightarrow E_{J}^{r}\right]$ ensemble for $J \geq 3$. Let us denote the free distance of the $i$ th code by $d_{i}, i=1,2, \ldots, J-$ 1. (Note that the free distance of the inner code $C_{J}$ plays no role; we only require that $E_{J}$ be recursive.) Now define

$$
\begin{aligned}
& \beta_{W}^{(J)}=\left\lceil\frac{d_{J-1}}{2}\right\rceil+\sum_{i=1}^{J-2}\left(d_{i}-1\right)-1 \\
& \beta_{b}^{(J)}=\left\lceil\frac{d_{J-1}}{2}\right\rceil+\sum_{i=1}^{J-2}\left(d_{i}-1\right) .
\end{aligned}
$$

Theorem 8.7: For the $\left[E_{1} \Rightarrow E_{2} \Rightarrow \cdots \Rightarrow E_{J}^{r}\right]$ ensemble, for $J \geq 3$, there exists a positive number $c_{0}$ such that for any 
binary-input memoryless channel whose noise exponent $\alpha$ satisfies $\alpha>c_{0}$

$$
\begin{aligned}
& \bar{P}_{W}^{(n)}=O\left(n^{-\beta_{W}^{(J)}+\epsilon}\right) \\
& \bar{P}_{b}^{(n)}=O\left(n^{-\beta_{b}^{(J)}+\epsilon}\right)
\end{aligned}
$$

for arbitrary $\epsilon>0$, where $\beta_{W}^{(J)}$ and $\beta_{b}^{(J)}$ are defined in (8.2) and (8.3).

The multiple serial ensembles with $J=3$ were considered by Benedetto et al.in [5], and their calculation of the corresponding interleaving gain exponent agrees with our formulas (8.2) and (8.3) for $J=3$.

\section{EXAMPLES}

It is interesting to consider the CCSDS "standard" $R=1 / 3$ turbo code [7] in the light of our results. This turbo code is a parallel concatenation with $J=2$ recursive convolutional component codes, $R_{1}=1 / 2, R_{2}=1$, and overall rate $R=1 / 3$. The two encoders are described by the transfer functions

$$
\begin{aligned}
& G_{1}(D)=\left(1, \frac{1+D+D^{3}+D^{4}}{1+D^{3}+D^{4}}\right) \\
& G_{2}(D)=\frac{1+D+D^{3}+D^{4}}{1+D^{3}+D^{4}} .
\end{aligned}
$$

Experimental evidence, together with density evolution analysis [11], with this ensemble on the additive white Gaussian noise (AWGN) channel suggests that for any value of $E_{b} / N_{0}$ greater than around $-0.05 \mathrm{~dB},{ }^{7}$ the bit error probability can be made arbitrarily small, in approximately inverse proportion to the block size, but the word error probability does not go to zero. If we apply Theorem 8.1 to this same ensemble, we get no quantitative information about the noise threshold, but we find that above the threshold, we have (ignoring the " $+\epsilon$ " in the exponent) $\bar{P}_{b}^{(n)}=$ $O(1 / n)$, and $\bar{P}_{W}^{(n)}=O(1)$, in gratifying agreement with experiment. It is important to bear in mind, however, that: 1) the experiments are with suboptimum iterative decoding, whereas Theorem 8.1 deals with MLD; 2) Theorem 8.1 only provides an upper bound on code performance, and does not preclude the possibility that a more rapid decrease in decoder error probability is possible; and 3) experiments always deal with particular interleavers, whereas Theorem 8.1 treats the average over all interleavers.

The repeat-accumulative (RA) codes introduced in [13] are serial turbo code ensembles with an $R_{1}=1 / q q$-fold repetition code as the outer code, and an $R_{2}=1$ recursive convolutional code, with transfer function $1 /(1+D)$, as the inner code. The outer code has minimum distance $d_{1}=q$. Hence, by Theorem 8.4 , on all memoryless binary input channels, RA codes have word error probability approaching zero for $q \geq 3$ and bit error probability approaching zero for $q \geq 2$. For this ensemble, we can say something quantitative about the noise thresholds, since we can compute the exact spectral shape [13]

$$
\begin{aligned}
r(\delta)=\max _{0 \leq x \leq 1 / q}\{- & \frac{q-1}{q} H(q x) \\
& \left.\quad+(1-\delta) H\left(\frac{q x}{2(1-\delta)}+\delta H\left(\frac{q x}{2 \delta}\right)\right)\right\} .
\end{aligned}
$$

${ }^{7}$ The Shannon limit for $R=1 / 3$ codes on the AWGN channel is $-0.495 \mathrm{~dB}$.
TABLE I

RA Ensemble Noise Thresholds, Quoted as Channel Crossover PROBABILITIES, OBTAINED FROM THE UNION BOUND (UB) AND THE "TYPICAL PAIRS" (TP) TECHNIQUES ON THE BSC. THE SHANNON LIMIT FOR THE ENSEMble of All Linear CODES of RATE $R$ Is Also Given

\begin{tabular}{lllll}
\hline$q$ & $R$ & UB & TP & Shannon Limit \\
3 & $1 / 3$ & 0.091 & 0.132 & 0.174 \\
4 & $1 / 4$ & 0.132 & 0.191 & 0.215 \\
5 & $1 / 5$ & 0.163 & 0.228 & 0.243 \\
6 & $1 / 6$ & 0.187 & 0.254 & 0.265 \\
7 & $1 / 7$ & 0.207 & 0.274 & 0.281 \\
\hline
\end{tabular}

TABLE II

RA ENSEMble NoISE THRESholds, QuOTEd AS $E_{b} / N_{0}$ (IN dB), OBTAINED FROM THE UNION BOUND (UB) AND THE "TYPICAL PAIRS" (TP) TECHNIQUES ON THE AWGN CHANNEL. The SHANNON Limit For THE ENSEMble OF AlL LINEAR CODES OF RATE $R$ IS ALSO GIVEN

\begin{tabular}{lllll}
\hline$q$ & $R$ & UB & TP & Shannon Limit $(\mathrm{dB})$ \\
3 & $1 / 3$ & 2.20 & 0.739 & -0.495 \\
4 & $1 / 4$ & 1.93 & -0.078 & -0.794 \\
5 & $1 / 5$ & 1.80 & -0.494 & -0.963 \\
6 & $1 / 6$ & 1.72 & -0.742 & -1.071 \\
7 & $1 / 7$ & 1.67 & -0.905 & -1.150 \\
\hline
\end{tabular}

Two short tables of these thresholds, on the binary-symmetric channel and the Gaussian channel respectively, are given next.

In Table I, the noise threshold is given as the largest value of the channel crossover probability for which the union bound guarantees good code performance for the corresponding RA ensemble. In Table II, the threshold is given as the smallest value of $E_{b} / N_{0}$ for which the union bound guarantees good performance. If the union bound is replaced with a more powerful tool, these thresholds can be considerably improved. For example, using the "typical pairs" method, we can obtain the "TP" column of Table I for RA codes on the BSC [1], and in the "TP" column of Table II for the AWGN channel [20].

\section{Discussion AND CONCLUSION}

The results in this paper are in a sense the culmination of a series of earlier papers [1], [10], [12], [13], [18]-[20]. In those papers, we were interested in computing channel noise thresholds for specific code ensembles on specific channels; in this paper, we have considered general ensembles on general channels. However, we have paid a price for this generality: whereas in the earlier papers our estimates for the noise thresholds were computed numerically, in this paper we only prove the existence of the thresholds. To get good numerical thresholds using our methodology would require at least two improvements. First, we would have to replace the union bound with a more powerful technique; and second, we would need much more accurate estimates for the asymptotic weight spectrum $r(\delta)$ of the ensembles in question.

We have already addressed the first of these two problems. In [1], [10], [12], [13], and [18] we have developed a tool, the "typical pairs" method, which is capable of reproducing Shannon's theorem for the ensemble of random linear codes. (Examples of the thresholds obtainable using these techniques are given in Tables I and II.) Additionally, the recent techniques of Divsalar [9], 
Duman and Salehi [16], [15], and Sason and Shamai [28], [32], [29], [30], which build on Gallager's technique [17], are all potentially capable of producing far stronger results than possible using the union bound.

However, these methods, despite their power, are useless unless one has an exact or near-exact expression for the asymptotic weight spectrum $r(\delta)$ of the ensemble in question. This is the second, and more difficult, of the needed improvements. To date, we can give good estimates for $r(\delta)$ in only three cases: the ensemble of all linear codes of rate $R($ here $r(\delta)=H(\delta)-(1-R)$ ), the ensemble of Gallager $(j, k)$ low-density parity-check codes [17], and the ensemble of RA codes [1]. A method for computing $r(\delta)$ for other ensembles, in particular the turbo code ensembles, would be very welcome. The recent results of Sason, Teletar, and Urbanke [31] may prove to be helpful in this direction.

Our main results provide only upper bounds on $\bar{P}_{W}^{(n)}$ and $\bar{P}_{b}^{(n)}$, but based on experimental evidence we conjecture that these bounds are close to best possible, viz., for any channel with $\gamma<\gamma_{0}, \bar{P}_{W}^{(n)}=\Omega\left(n^{-\beta}\right){ }^{8}$ More generally, for any binary-input discrete memoryless channel, we conjecture that for any value of $\gamma$, either

$$
\lim _{n \rightarrow \infty} \bar{P}_{W}^{(n)}=1
$$

or

$$
\bar{P}_{W}^{(n)}=\Theta\left(n^{-\beta}\right) .
$$

If these conjectures are true, it follows that the interleaving gain exponent $\beta$ is an important measure of the ensemble's performance, and not just an artifact of our method of proof.

Finally, we mention the important alternative approach to this problem recently announced by Richardson and Urbanke [27]. This work extends their earlier, landmark work on low-density parity-check codes [25], and deals directly with the performance of iterative decoding. They show, for any $J=2$, rate $1 / 3$ parallel turbo ensemble, on a extensive class of symmetric binary-input channels, the existence of a noise threshold $\sigma^{*}$, such that if the noise is below $\sigma^{*}$, the ensemble bit error probability can be made arbitrarly small, whereas if the noise exceeds $\sigma^{*}$, the ensemble bit error probability is bounded away from zero. Furthermore, they describe a numerical algorithm that can be used to find the exact value of $\sigma^{*}$ in many cases. In many ways, this work surpasses ours for the (ensemble, channel) pairs to which it applies. The only pieces of our main results apparently not present in $R-U$ is quantitative information about the rate at which $\bar{P}_{b}^{(n)}$ approaches zero, and information about the word error probability. We conjecture that the $R-U$ analysis can be extended to the general $\left[E_{1}^{r}\left\|E_{2}^{r}\right\| \cdots \| E_{J}^{r}\right]$ and $\left[E_{1} \Rightarrow E_{2}^{r}\right]$ ensembles, and to all memoryless binary-input channels.

\section{APPENDIX A}

\section{Combinatorial Facts About TRuncATED Convolutional Codes}

In this appendix, we shall state for reference three useful combinatorial facts about the weight structure of convolutional

${ }^{8}$ Recall that $f(n)=O(g(n))$ means that $f(n) \leq K_{1} g(n)$, for some constant $K_{1}, f(n)=\Omega(g(n))$ means that $f(n) \geq K_{2} g(n)$, for some constant $K_{2}$, and $f(n)=\Theta(g(n)$ means that $f(n)=O(g(n))$ and $f(n)=\Omega(G(n))$. codes, due essentially to Kahale and Urbanke [21]. (Although Theorems A.2 and A.3 were stated in [21] only for systematic rate $1 / 2$ codes, the proofs given apply in the generality we state.)

Theorem A.l (The $\eta-\mu$ Theorem): For a noncatastrophic convolutional encoder $E$, there exists a constant $\mu, \mu=\mu(E)$, such that if the output weight is $h$, then the input weight is at most $\mu h$. Also, there is a constant $\eta=\eta(E)$ such that if a codeword in the truncated code consists of several detours, of total length $L_{0}$, then the codeword weight $d$ satisfies $d \geq L_{0} \eta$.

In what follows, $A_{h}^{(L)}$ denotes the number of codewords of weight $h$ in the $L$ th truncation of the code and $A_{w, h}^{(L)}$ denotes the corresponding number of codewords with input weight $w$ and output weight $h$. Thus,

$$
A_{h}^{(L)}=\sum_{w=1}^{k} A_{w, h}^{(L)}=\left(\text { by Theorem A.1) }=\sum_{w=1}^{\mu h} A_{w, h}^{(L)} .\right.
$$

Similarly, $A_{w, \leq h}^{(L)}$ denotes the number of codewords with input weight $w$ and output weight less than or equal to $h$, i.e.,

$$
A_{w, \leq h}^{(L)}=\sum_{d=1}^{h} A_{w, d}^{(L)} .
$$

Theorem A.2 (cf. [21, Lemma 3]): Let $C$ be an $(n, k, m)$ convolutional code, as represented by a noncatastrophic encoder $E$. Then for the $(n L, k L-m)$ block code obtained by truncating $C$ at depth $L$

$$
A_{h}^{(L)} \leq \theta^{h}\left(\begin{array}{c}
L \\
\left\lfloor h / d_{1}\right\rfloor
\end{array}\right)
$$

where $d_{1}$ is the free distance of the code, and $\theta \eta$ is a constant independent of $h$ and $n$.

We define a recursive convolutional code to be one for which any input of weight 1 produces an output of infinite weight.

Theorem A.3 (cf. [21, Lemma 1]): Let $C$ be an $(n, k, m)$ recursive convolutional code, with corresponding noncatastrophic encoder $E$. Then, for the $(n L, k L-m)$ block code obtained by truncating the $E$-trellis representation of $C$ at depth $L$

$$
A_{w, \leq h}^{(L)} \leq \theta^{w} \sum_{j=0}^{\lfloor w / 2\rfloor}\left(\begin{array}{c}
L \\
j
\end{array}\right)\left(\begin{array}{c}
\eta h \\
w-j
\end{array}\right)
$$

where $\theta \eta$ and $\eta$ are constants independent of $w, h$, and $n$. (For the significance of $\eta$, see Theorem A.1.)

APPENDIX B SOME USEFUL INEQUALITIES

Suppose $n, k$ are positive integers, $1 \leq k \leq n$. Then

$$
\begin{aligned}
\left(\begin{array}{l}
n \\
k
\end{array}\right) & \leq 2^{n} \\
\frac{n^{k}}{k^{k}} & \leq\left(\begin{array}{l}
n \\
k
\end{array}\right) \leq n^{k} \\
e^{n H(k / n)} /(n+1) & \leq\left(\begin{array}{l}
n \\
k
\end{array}\right) \leq e^{n H(k / n)} .
\end{aligned}
$$

(For (B3), see [8, Example 12.1.3, p. 284].) 
Proposition B.1: If $n \geq m, 0 \leq j \leq\lfloor w / 2\rfloor$, then

$$
\begin{aligned}
\left(\begin{array}{c}
n \\
j
\end{array}\right)\left(\begin{array}{c}
m \\
w-j
\end{array}\right) & \leq\left(\begin{array}{c}
n \\
\lfloor w / 2\rfloor
\end{array}\right)\left(\begin{array}{c}
m \\
w-\lfloor w / 2\rfloor
\end{array}\right) \\
& =\left(\begin{array}{c}
n \\
\lfloor w / 2\rfloor
\end{array}\right)\left(\begin{array}{c}
m \\
\lceil w / 2\rceil
\end{array}\right) .
\end{aligned}
$$

Proof: It suffices to show that $f(j)=\left(\begin{array}{c}n \\ j\end{array}\right)\left(\begin{array}{c}m \\ w-j\end{array}\right)$ is an increasing function of $j$, for $0 \leq j \leq\lfloor w / 2\rfloor$. Consider the ratio

$$
\frac{f(j)}{f(j-1)}=\frac{n-j+1}{m-w+j} \frac{w-j+1}{j}, \quad \text { for } j \geq 1
$$

since $w-j+1 \geq j$ and $n-j+1 \geq m-w+j$, we have $f(j) / f(j-1) \geq 1$. Hence the conclusion follows.

\section{Proposition B.2:}

1) Given

$$
F_{n}(w)=\Theta^{w} n^{J\lfloor w / 2\rfloor-(J-1) w} D_{n}^{(2 J-1) w}, \quad 1 \leq w \leq \mu D_{n}
$$

$F_{n}(2)$ will be the largest term as $n$ becomes large.

2) Given

$$
G_{n}(d)=\Theta^{d} n^{\left\lfloor d / d_{1}\right\rfloor+\lfloor d / 2\rfloor-d} D_{n}^{2 d}, \quad d_{1} \leq d \leq \mu D_{n}
$$

$G_{n}\left(d_{1}\right)$ will be the largest term as $n$ becomes large. Proof:

1) It is easy to show that $F_{n}(w)$ satisfies

$$
F_{n}(1) \geq F_{n}(3) \geq F_{n}(5) \geq \cdots
$$

and

$$
F_{n}(2) \geq F_{n}(4) \geq F_{n}(6) \geq \cdots
$$

as $n$ gets large by taking the ratio of two consecutive terms. Verifying that $F_{n}(2) \geq F_{n}(1)$ for large $n$, we have the claim.

2) Similarly, we can show

$$
G_{n}\left(d_{1}\right) \geq G_{n}\left(d_{1}+1\right) \geq \cdots \geq G_{n}\left(\mu D_{n}\right)
$$

by taking the ratio of two consecutive terms.

Proposition B.3: Given real numbers $\alpha_{i}, \beta_{i}$ for $i=1, \ldots, n$, with $\beta_{i} \geq 0$, define

$$
\Delta=\sum_{i=1}^{n} \alpha_{i} \beta_{i}
$$

and for $\mu>0$, let

$$
L=\lim _{\delta \rightarrow 0} \frac{1}{\delta}\left(\sup _{0<x<\mu \delta} \sum_{i=1}^{n} \alpha_{i} H\left(\beta_{i} x\right)\right) .
$$

Then $L<+\infty$, if $\Delta \leq 0$.

Proof (Sketch): It is easy to see that for small $x$

$$
H(x)=x \log \frac{1}{x}+x+O\left(x^{2}\right)
$$

and so

$$
\begin{array}{rl}
\sum_{i} \alpha_{i} & H\left(\beta_{i} x\right) \\
& =\Delta x \log \frac{1}{x}+\left(\sum_{i} \alpha_{i} \beta_{i}\left(1+\log \frac{1}{\beta_{i}}\right)\right) x+O\left(x^{2}\right) .
\end{array}
$$

If $\Delta<0$, the first term in the above expansion dominates, and the result follows immediately (indeed, the limit is 0 ). If $\Delta=0$ we have

$$
\sum_{i} \alpha_{i} H\left(\beta_{i} x\right)=\left(\sum_{i} \alpha_{i} \beta_{i} \log \left(\frac{1}{\beta_{i}}\right)\right) x+O\left(x^{2}\right)
$$

in which case the "sup" in (B4) is attained at $x=\mu \delta$ as $\delta \rightarrow 0$, and the limit is finite.

\section{APPENDIX C}

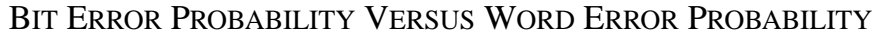

The union bound on the bit error probability for MLD of an $(n, k)$ binary linear code $C$ with IOWE $\left(A_{w, k}\right)$ over a memoryless binary input channel has the following form:

$$
P_{b} \leq \sum_{h=1}^{n} \sum_{w=1}^{k} \frac{w}{k} A_{w, h} e^{-\alpha h} .
$$

In this appendix, we will state, and sketch a proof of, a theorem on the ensemble bit error probability $\bar{P}_{b}^{(n)}$, analogous to Theorem 5.1 (which deals with word error probability). To that end, we define another innominate sum

$$
Y^{(n)}(D) \triangleq \sum_{h=1}^{D} \sum_{w=1}^{k} \frac{w}{k} \bar{A}_{w, h}^{(n)}
$$

Theorem C.1: If the threshold $c_{0}$ defined in (5.4) is finite, then if $\alpha>c_{0}$, there exists an integer $n_{0}$ and positive constants $K$ and $\epsilon$ such that for $n \geq n_{0}$

$$
\bar{P}_{b}^{(n)} \leq Y^{(n)}\left(D_{n}\right)+K e^{-\epsilon D_{n}} .
$$

Proof (Sketch): Beginning with (C1), we have

$$
\begin{aligned}
\bar{P}_{b}^{(n)} & \leq \sum_{h=1}^{n} \sum_{w=1}^{k} \frac{w}{k} \bar{A}_{w, h}^{(n)} e^{-\alpha h} \\
& \leq \sum_{h=1}^{D} \sum_{w=1}^{k} \frac{w}{k} \bar{A}_{w, h}^{(n)}+\sum_{h>D} \sum_{w=1}^{k} \frac{w}{k} \bar{A}_{w, h}^{(n)} e^{-\alpha h} \\
& =Y^{(n)}(D)+\sum_{h>D} \sum_{w=1}^{k} \frac{w}{k} \bar{A}_{w, h}^{(n)} e^{-\alpha h} \\
& \leq Y^{(n)}(D)+\sum_{h>D} \sum_{w=1}^{k} \bar{A}_{w, h}^{(n)} e^{-\alpha h} \\
& =Y^{(n)}(D)+\sum_{h>D} \bar{A}_{h}^{(n)} e^{-\alpha h} .
\end{aligned}
$$

Theorem C.1 now follows almost immediately from (C4) and the proof of Theorem 5.3.

Corollary C.2: If in addition, $Y^{(n)}\left(D_{n}\right)=O\left(n^{-\beta}\right)$, where $\beta>0$, then for $\alpha>c_{0}$

$$
\bar{P}_{b}^{(n)}=O\left(n^{-\beta}\right)
$$

The following lemma shows how the results on word error probability can be easily extended to bit error probability. In essence, Lemma C.3 shows that $Z^{(n)}\left(D_{n}\right)=O\left(n^{-\beta}\right)$ if and only if $Y^{(n)}\left(D_{n}\right)=O\left(n^{-\beta+1}\right)$.

Lemma C.3: There exists a positive constant $\mu$, such that

$$
Z^{(n)}\left(D_{n}\right) / k \leq Y^{(n)}\left(D_{n}\right) \leq \mu D_{n} Z^{(n)}\left(D_{n}\right) / k \text {. }
$$


Proof: Applying $w / k \geq 1 / k$ to (C2), we obtain the left inequality. From Proposition A.1 we know that if $\bar{A}_{w, h}^{(n)} \neq 0$, then $w \leq \mu h$. Thus, if $h \leq D_{n}$, and $\bar{A}_{w, h}^{(n)} \neq 0$, then $w \leq \mu h \leq \mu D_{n}$. The right-hand inequality then follows if we upper bound $w / k$ by $\mu D_{n} / k$ in (C2). Finally, since $k=R n$, where $R$ is the rate of the ensemble, it follows that $Z^{(n)}\left(D_{n}\right)=O\left(n^{-\beta}\right)$ iff $Y^{(n)}\left(D_{n}\right)=O\left(n^{-\beta+1}\right)$.

\section{ACKNOWLEDGMENT}

The authors would like to thank Dariush Divsalar, who did the density evolution analysis for the $R=1 / 3$ CCDSD code, and Rüdiger Urbanke for assuring us that the bounds in Appendix A indeed follow from the results in [21].

\section{REFERENCES}

[1] S. M. Aji, H. Jin, D. MacKay, and R. J. McEliece, "BSC thresholds for code ensembles based on 'Typical Pairs' decoding," in Proc. IMA Workshop Codes and Graphs, "Codes, Systems, and Graphical Models", Minneapolis, MN, Aug. 1999, pp. 195-210.

[2] S. Benedetto and G. Montorsi, "Unveiling turbo codes: Some results on parallel concatenated coding schemes," IEEE Trans. Inform. Theory, vol. 42, pp. 409-428, Mar. 1996.

[3] — "Design of parallel concatenated convolutional codes," IEEE Trans. Commun., vol. 44, pp. 591-600, May 1996.

[4] S. Benedetto, D. Divsalar, G. Montorsi, and F. Pollara, "Serial concatenation of interleaved codes: Performance analysis, design, and iterative decoding," IEEE Trans. Inform. Theory, vol. 44, pp. 909-926, May 1998.

[5] - "Analysis, design, and iterative decoding of double serially concatenated codes with interleavers," IEEE J. Select. Areas of Commun. vol. 16, pp. 231-244, Feb. 1998.

[6] C. Berrou, A. Glavieux, and P. Thitimajshima, "Near Shannon limit error-correcting coding and decoding: Turbo codes," in Proc. 1993 IEEE Int. Conf. Communications, Geneva, Switzerland, May 1993, pp. 1064-1070.

[7] Consultative Committee for Space Data Systems (CCSDC), "Telemetry channel coding," Blue Book, no. 4, May 1999. [Online]. Available: http://www.ccsds. org.documents/pdf/CCSDS-101.0-B-4.pdf.

[8] T. M. Cover and J. A. Thomas, Elements of Information Theory. New York: Wiley, 1991.

[9] D. Divsalar. A simple tight bound on error probability of block codes with application to turbo codes. TDA Progr. Rep., vol. 42-139, July-Sept. 1999. [Online]. Available: http://tmo.jpl.nasa.gov/tmo/ progress_report/42-139/139L.pdf.

[10] D. Divsalar, S. Dolinar, H. Jin, and R. McEliece, "AWGN coding theorems from ensemble weight enumerators," in Proc. IEEE Int. Symp. Information Theory, Sorrento, Italy, June 2000, p. 458

[11] D. Divsalar, S. Dolinar, and F. Pollara, "Iterative turbo decoder analysis based on density evolution," IEEE J. Select. Areas Commun., vol. 19, pp. 891-907, May 2001.

[12] D. Divsalar and R. J. McEliece. On the design of concatenated coding systems with interleavers. JPL TMO Progr. Rep., vol. 2-134, pp. 1-22, Aug. 15, 1998. [Online]. Available: http://tmo.jpl.nasa.gov/tmo/ progress_report/42-134/134D.pdf.
[13] D. Divsalar, H. Jin, and R. McEliece, "Coding theorems for "TurboLike' codes," in Proc. 1998 Allerton Conf. Communications, Control and Computing, Monticello, IL, pp. 201-210.

[14] D. Divsalar and F. Pollara, "On the design of turbo codes," TDA Progr. Rep., vol. 42-123, pp. 99-121, Nov. 15, 1995.

[15] T. M. Duman, "Turbo codes and turbo coded modulation systems: Analysis and performance bounds," Ph.D. dissertation, ECE Dept., Northeastern Univ., Boston, MA, May 1998.

[16] T. M. Duman and M. Salehi, "New performance bounds for turbo codes," IEEE Trans. Commun., vol. 46, pp. 717-723, June 1998.

[17] R. Gallager, Low-Density Parity-Check Codes. Cambridge, MA: MIT Press, 1963

[18] H. Jin and R. J. McEliece, "AWGN coding theorems for serial turbo codes," in Proc. 37th Allerton Conf. Communication, Computation and Control, Allerton, IL, Sept. 1999, pp. 893-894.

[19] — - "RA codes achieve AWGN channel capacity," in Proc. 13th Int. Symp. AAECC-13 (Lecture Notes in Computer Science). New York: Springer-Verlag, 1999, pp. 10-18.

[20] - "Typical pairs decoding on the AWGN channel," in Proc. 2000 Int. Symp. Information Theory and Its Applications, pp. 180-183.

[21] N. Kahale and R. Urbanke, "On the minimum distance of parallel and serially concatenated codes," IEEE Trans. Inform. Theory, submitted for publication.

[22] D. J. C. MacKay, "Good error-correcting codes based on very sparse matrices," IEEE Trans. Inform. Theory, vol. 45, pp. 399-431, Mar. 1999.

[23] R. J. McEliece, The Theory of Information and Coding. Reading, MA: Addison-Wesley, 1977. (2nd ed.: Cambridge, U.K.: Cambridge Univ. Press, 2002).

[24] R. J. McEliece, D. J. C. MacKay, and J.-F. Cheng, "Turbo decoding as instance of Pearl's 'Belief Propagation' algorithm," IEEE J. Select. Areas Commun., vol. 16, pp. 140-152, Feb. 1998.

[25] T. Richardson and R. Urbanke, "The capacity of low-density paritycheck codes under message-passing decoding," IEEE Trans. Inform. Theory, vol. 47, pp. 599-618, Feb. 2001.

[26] T. Richardson, A. Shokrollahi, and R. Urbanke, "Design of capacity-approaching irregular low-density parity-check codes," IEEE Trans. Inform. Theory, vol. 47, pp. 619-637, Feb. 2001.

[27] T. Richardson and R. Urbanke, "Thresholds for turbo codes," in Proc. IEEE Int. Symp. Information Theory, Sorrento, Italy, June 2000, p. 317

[28] I. Sason and S. Shamai (Shitz), "On improved bounds on coded communications over interleaved fading channels, with application to turbo codes," in Proc. 2nd Int. Symp. Turbo Codes and Related Topics, Brest, France, Sept. 4-7, 2000, pp. 239-243.

[29] — - "On improved bounds on the decoding error probability of block codes over interleaved fading channels, with applications to turbo-like codes," IEEE Trans. Inform. Theory, vol. 47, pp. 2275-2299, Sept. 2001.

[30] — , "On Gallager-type bounds for the mismatched decoding regime with applications to turbo codes," in Proc. IEEE Int. Symp. Information Theory (ISIT 2001), Washington, DC, June 24-29, 2001, p. 134.

[31] I. Sason, E. Telatar, and R. Urbanke. The asymptotic input-output weight distribution of convolutional codes. presented at 38th Annual Allerton Conference on Communications, Control and Computing, Monticello, IL, Oct. 2000. [Online]. Available: http://thcwww.epfl.ch/ publications.html.

[32] S. Shamai (Shitz) and I. Sason, "Variations on Gallager's bounding techniques: Performance bounds for turbo codes in Gaussian and fading channels," in Proc. 2nd Int. Symp. Turbo Codes and Related Topics, Brest, France, September 4-7, 2000, pp. 27-34.

[33] C. E. Shannon, The Mathematical Theory of Information. Urbana, IL: Univ. Illinois Press, 1949. (Reprinted 1998). 